\title{
R Packages to Aid in Handling Web Access Logs
}

by Oliver Keyes, Bob Rudis, Jay Jacobs

\begin{abstract}
Web access logs contain information on HTTP(S) requests and form a key part of both industry and academic explorations of human behaviour on the internet. But the preparation (reading, parsing and manipulation) of that data is just unique enough to make generalized tools unfit for the task, both in programming time and processing time which are compounded when dealing with large data sets common with web access logs. In this paper we explain and demonstrate a series of packages designed to efficiently read in, parse and munge access log data, allowing researchers to handle URLs and IP addresses easily. These packages are substantially faster than existing $\mathrm{R}$ methods - from a $3-500 \%$ speedup for file reading to a $57,000 \%$ speedup in URL parsing.
\end{abstract}

\section{Introduction}

The rise of the World Wide Web has made it dramatically easier to access and transfer data, and $\mathrm{R}$ boasts abundant functionality when it comes to taking data from the web. Base R itself has simple file downloading and page reading capabilities, through the download. file and readLines functions, and additional functionality is made available for handling web-accessible data through packages such as httr (Wickham, 2015).

Data on the web is not, however, the only kind of web data that interests researchers; web traffic is, in and of itself, an interesting data source. Access logs-records of connections between users and a web server-are an asset and resource for people studying everything from user behaviour on the internet (Halfaker et al., 2014), to website performance (Ryckbosch and Diwan, 2014), to information security (Bhingarkar and Shah, 2015).

An example scenario for those users is the need to be able to read in access logs, parse the URLs to extract relevant metadata about the access requests, geolocate the people making those requests to understand how behaviour and desired content vary between populations.

As a statistically-oriented programming language, $\mathrm{R}$ is commonly used by these same researchers for data analysis, testing and reporting. However, the general-purpose functions within $\mathrm{R}$ require significant customization before they can handle the scenario above. Additionally, the off-the-shelf functions are inefficient for these tasks, especially when scaling to the large data sets that are common with web access logs. In this article we review the use cases for particular operations over web data, the limitations in base $\mathrm{R}$ when it comes to performing those operations, and a suite of $\mathrm{R}$ packages designed to overcome them: reading access logs in (webreadr), manipulating URLs (urltools), manipulating IP addresses (iptools), and direct IP geolocation (rgeolocate).

\section{Reading access logs}

The first task with any data analysis is to read the data into R. With access logs this is slightly complicated by the fact that there is no one standard for what a log should look like; instead, there are multiple competing approaches from different software platforms and eras. These include the Common Log Format (CLF), the confusingly-named Combined Log Format, and formats used by individual, commonly-used software platforms - such as the custom format for the Squid internet caching software, and the format used by Amazon Web Services (AWS).

One difference between formats can easily be shown by looking at how timestamps are represented:

Table 1: Timestamps in Common Access Log Formats

\begin{tabular}{lll}
\hline Log Type & Timestamp Columns & Timestamp Format \\
\hline Common Log Format & 1 & 10/Oct/2000:13:55:36 -0700 \\
Combined Log Format & 1 & 26/Apr/2000:00:23:48 -0400 \\
Squid & 1 & 1286536309.450 \\
AWS & 2 & $2014-05-23$ 01:13:11 \\
\hline
\end{tabular}

With four log types, we have three different timestamp formats, and timestamp is only one of the many columns that could appear. These logs also vary in whether they specify quoting fields (or sanitising unquoted ones), the columns they contain and the data each column contains in turn. 
Base $\mathrm{R}$ does not have a way of easily reading in any one of those formats, let alone all of them (understandably, given its statistical orientation). There is also the ApacheLogProcessor (Mendonca, 2015) package, but (although more dedicated than base R) it is only capable of reading Common Log Format files (although it does have novel features such as optional parallel processing for the data cleanup tasks).

To make reading access logs into $\mathrm{R}$ as easy as possible we created the webreadr (Keyes, 2015) package. This contains user-friendly equivalents to read. table for each type of log, detecting the fields that should appear, converting the timestamps into POSIX objects, and merging fields or splitting fields where necessary. The package contains four core functions, one for each form of log: read_clf for the Common Log Format, read_combined for the Combined Log Format, and read_squid and read_aws for Squid and AWS formats respectively. Each one abstracts away the complexity of specifying column names and formats, and instead allows a researcher to read a file in with a minimal amount of work: the only parameters that need to be specified are the path to the file, and whether the file has column headers.

As the name suggests, it is built not on top of base $\mathrm{R}$ but on top of the readr (Wickham and Francois) package, allowing us to take advantage of substantial speed improvements that package's base functions have over base R (ApacheLogProcessor, in contrast, relies on those same base functions). These improvements can be seen in the visualisation below, which uses microbenchmark (Mersmann, 2014) to compare 100 reads of a 600,000-line "squid" formatted file with webreadr to the same operation performed in base R:

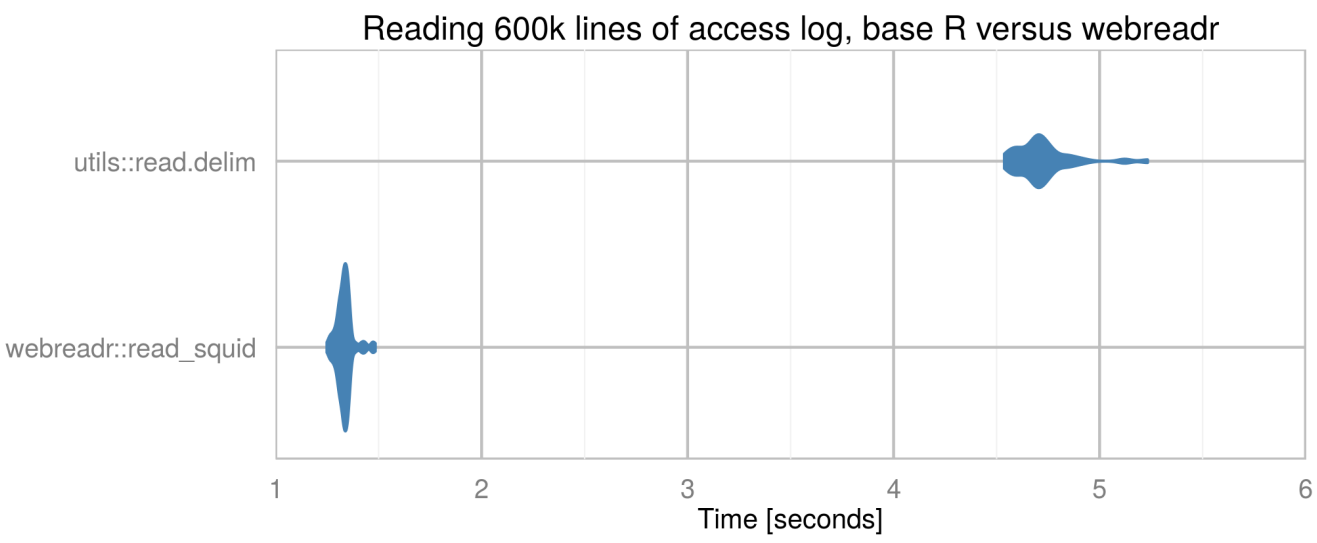

Figure 1: Results of microbenchmark run: read_squid versus base-R equivalent code

As this plot shows, webreadr is consistently 3.5-6 times faster than the equivalent base $\mathrm{R}$ functionality, and, as explained above, is also far simpler to use. ApacheLogProcessor was not benchmarked for the simple reason that it is built on the base R functionality tested, and so would be duplicative.

\section{Decoding and parsing URLs}

URLs are commonplace in access logs, describing both the web asset or page that the user requested, and the page the user came from. These fields are usually named url and referer respectively.

\section{Decoding}

Both values can be percent-encoded, allowing them to include characters that are valid but reserved by the URL specification as having special meanings ("reserved characters"). A '\#' symbol, for example, is encoded as '\%23': a percentage symbol, followed by a unique numerical value for that character.

The encoding of reserved characters is useful, since it means that URL paths and queries can contain a vast array of values - but it makes data analysis tougher to do. Examples of common data analysis or cleaning operations that become more difficult are:

1. Aggregation. Aggregating URLs together is useful to identify, for example, the relative usage and popularity of particular pages in your data - but it becomes tougher if encoding is inconsistent, because two URLs could hold the same value but look very different.

2. Value selection. With text-based data, regular expressions are a common way of filtering or selecting entries that meet particular conditions, but things become fuzzy when you have to look not just for particular characters (a space, say) but also the encoded values (\%20). 
3. Exploratory data analysis (EDA). EDA is a common initial step to investigate a data set, examining the variables and values it contains and whether they meet a researcher's expectations but on a practical basis it becomes difficult when the values aren't human-readable.

The solution is to be able to consistently decode URLs, which makes URL-based data far easier to analyse. Base R contains the function URLdecode for this purpose, but, as it is neither vectorised nor based on compiled code, it can be extremely slow over large datasets.

To solve this common problem in analysing request logs, the urltools (Keyes et al., 2015a) package was created. This contains a function, url_decode, which decodes URLs and relies on vectorised, compiled code to do so. Benchmarking the two approaches against each other shows that the urltools implementation is approximately 60-70 times faster over large datasets. Again using microbenchmark, if we compare the vectorised decoding of 1,000,000 URLs with urltools against a URLdecode vapply loop, we see a 60-70 times speed improvement:

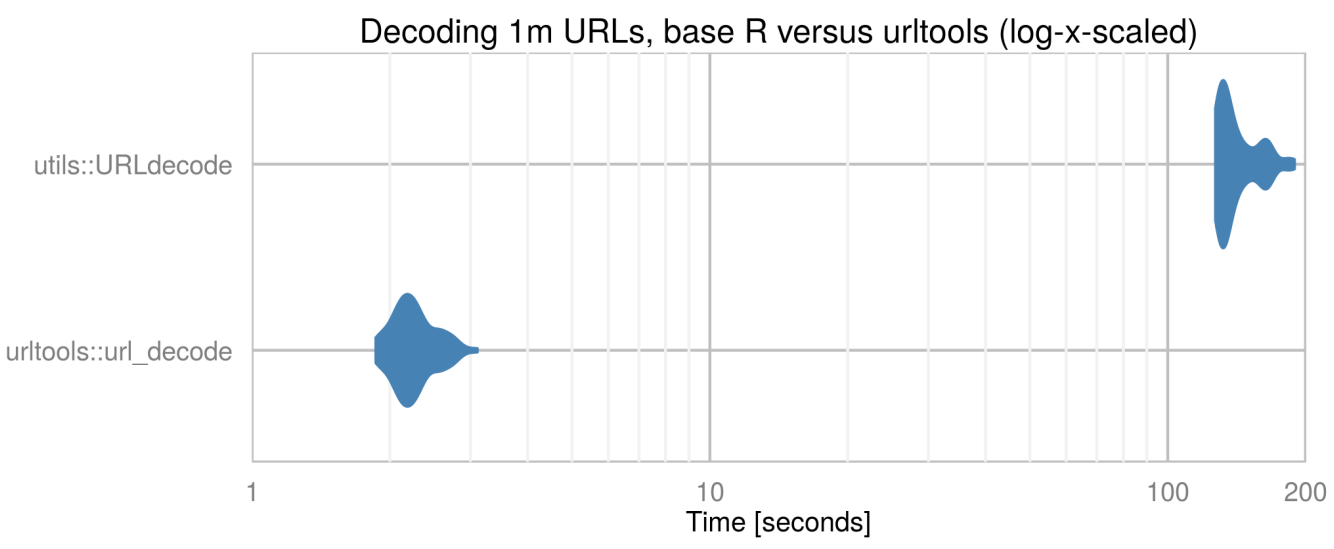

Figure 2: Results of microbenchmark run: url_decode versus base-R equivalent code

\section{Parsing}

The standard for URLs (Berners-Lee et al., 1994) divides them into a heirarchical sequence of components - the scheme ('http'), host ('en.wikipedia.org'), port ('800'), path ('wiki/Main_Page') and searchpart, or query string ('action=edit'). Together, these make up a URL ('http://en.wikipedia.org:800/wiki/Main_Page?action=edit').

Parsing URLs to isolate and extract these components is a useful ability when it comes to exploring request logs; it lets a researcher pick out particular schemes, paths, hosts or other components to aggregate by, identifying how users are behaving and what they are visiting. It makes anonymising data - by removing, for example, the parameters or path, which can contain relatively unique information easier.

Base R does not have native code to parse URLs, but the httr package (Wickham, 2015) contains a function, parse_url, designed to do just that. Built on R's regular expressions, this function is not vectorised, does not make use of compiled code internally, and produces a list rather than data.frame, making looping over a set of URLs to parse each one a time-consuming experience. This is understandable given the intent behind that function, which is to decompose individual URLs within the context of making HTTP requests, rather than to analyse URLs en masse. Similarly, the XML (Lang and the CRAN Team, 2016) package has parseURI; C-based, this time, but both dependent on the libxml library and, similarly, not vectorised.

urltools contains url_parse - which does the same thing as the equivalent httr functionality, but in a vectorised way, relying on compiled code, and producing a data. frame. Within the context of parsing and processing access logs, this is far more useful, because it works efficiently over large sets: httr's functionality, which was never designed with vectorisation in mind, does not. Indeed, benchmarking showed that url_parse is approximately 570 times faster than httr's equivalent function: 


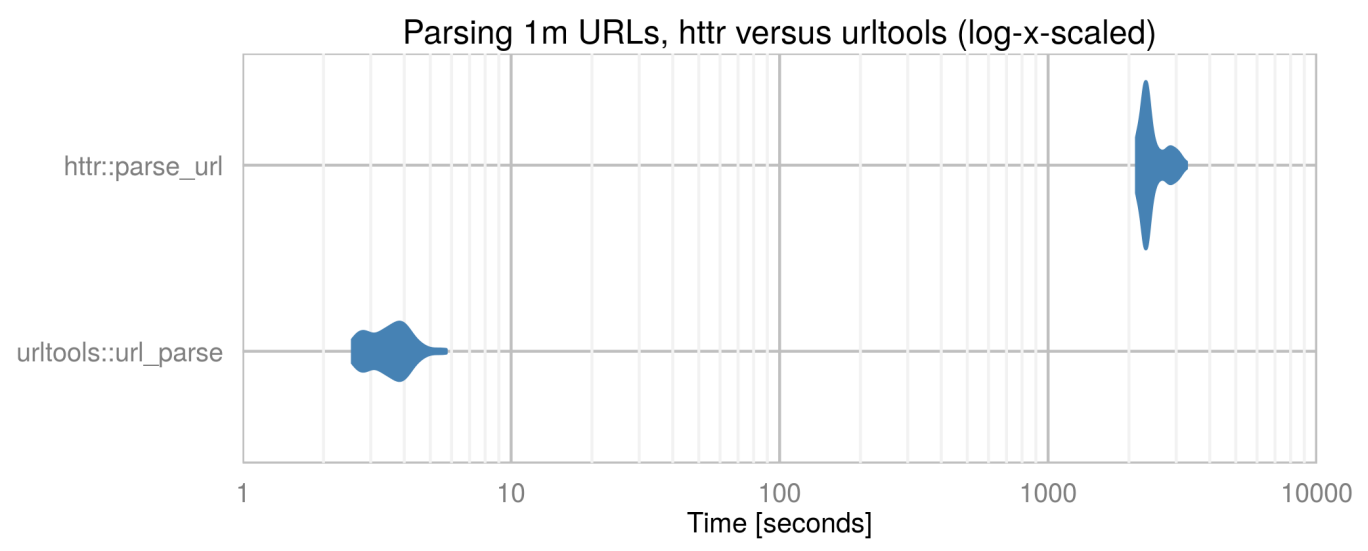

Figure 3: Results of microbenchmark run: url_parse versus httr's equivalent code

A vector of URLs passed into url_parse produces a data.frame, with one column for each of the IETF-supported components, and empty strings representing components that could not be found. Additionally, influenced by the style of the lubridate package (Grolemund and Wickham, 2011), urltools contains functions to get or set individual components:

\# Load urltools and construct a URL

library(urltools)

url <- "http://www.google.com/"

\# Get the scheme

scheme(url)

\#> [1] "http"

\# Set the scheme and observe the modification to the resulting URL scheme(url) <- "https"

url

\#> [1] "https://www.google.com/"

As a result of this functionality, urltools makes URL manipulation faster, easier and far more accessible, reducing the burden associated with munging access logs or similar datasets and allowing a researcher to get to the statistical analysis faster.

\section{IP manipulation}

Access logs also contain IP addresses - unique numeric values that identify a particular computer or network in the context of the internet. Working with these values allows an analyst to validate their data (by checking for false or spoof addresses) and is a necessary prerequisite to the use of some IP geolocation systems (covered later), and extract a limited amount of metadata from the values themselves.

iptools (Rudis and Keyes, 2015), based around the Boost ASIO C++ library (boo), is a package designed for this kind of IP manipulation (and more). Built around combined code, it is extremely fast (for that code that does not rely on internet connections) and boasts a range of features.

One of the most crucial is ip_classify, which, when provided with a vector of IP addresses, identifies whether they follow the IPv4 or IPv6 standard. As a side-effect of this, it can be used to identify if IP addresses are invalid, or spoofed, prior to further work based on the assumption that they are correct:

\# Load iptools and construct a vector of IPs

library(iptools)

ip_addresses <- c("192.168.0.1", "2607:f8b0:4006:80b::1004", "Chewie")

ip_classify(ip_addresses)

\#> [1] "IPv4" "IPv6" "Invalid" 
Along with this general tool there are also functions for ascertaining specific facts about IP addresses, returning logical (TRUE or FALSE) values. These are is_valid, is_ipv4, is_ipv6, which are built on top of ip_classify, and is_multicast, which identifies if an IP address is multicast designed to point to multiple machines.

iptools also contains (for similar purposes) code to identify the actual client's IP address. Access requests usually contain not only an IP address but a $X$-Forwarded-For field - a field identifying which other IP addresses the request passed through, if the user who made the request is using some kind of proxy. If a user has used a proxy, the contents of the IP address field won't actually be them - it will be the last proxy the request went through before getting to the server logging the requests. The actual IP address of the user will instead be the earliest value of the X-Forwarded-For field.

The solution is to be able to identify the 'real' IP address, by checking:

1. Whether the X-Forwarded-For field contains any values;

2. Extracting the earliest non-invalid IP address in that field's values if so, and the contents of the IP address field if not.

With the xff_extract function, you can do just that:

ip_address <- "192.168.0.1"

x_forwarded_for <- "foo, 193.168.0.1, 230.98.107.1"

xff_extract(ip_address, x_forwarded_for)

\#> [1] "192.168.0.1"

This returns the IP address field value if X-Forwarded-For is empty, and otherwise splits the X-Forwarded-For field and returns the earliest valid IP address. It is fully vectorised and highly useful for analysis predicated on IP addresses being valid, such as geolocation - without this kind of resolution, what you're actually geolocating might be your own servers.

Other operations supported by iptools include the conversion of IP addresses from their standard dotted-decimal form to a numeric form, the extraction of IP ranges (and their contents), resolving IP addresses to hostnames, and a series of datasets covering the IPv4 registry and port database.

The limitation of the package is that some operations do not yet support IPv6 (since they require the storage of numbers bigger than $\mathrm{R}$ can currently handle).

\section{Geolocation}

As a side-effect of how IP addresses tend to be assigned - in geographic blocks, to individual machines or to local networks - they can be used to geolocate requests, identifying where in the world the request came from, sometimes down to the level of individual post codes or pairs of latitude/longitude coordinates.

This is tremendously useful in industry, where the geographic reach of a service has substantial implications for its viability and survivability, and in academia, where the locality of internet-provided information and the breadth of internet access are active concerns and areas of study (Sen et al., 2015).

Many services and databases exist for extracting geographic metadata from IP addresses. One of the most common is the service provided by MaxMind, which has both proprietary and openlylicensed databases, in binary and comma-separated formats. The free databases have been used by various web APIs, which makes the data they contain accessible from R. Unfortunately, dependence on web APIs means that handling large numbers of IP addresses can be very slow (they tend to be designed to only accept one IP address at a time, and may contain throttling beyond that) and has privacy concerns, since it essentially means sending user IP addresses to a third party. And even without these issues, there are no wrappers for those APIs available on CRAN: users have to write their own.

With these concerns in mind, we wrote the rgeolocate (Keyes et al., 2015b) package. Through httr this contains convenient, vectorised bindings to various web services that provide geographic metadata about IP addresses. More importantly, using the Rcpp package to integrate $\mathrm{C}++$ and $\mathrm{R}$, rgeolocate also features a direct, compiled binding to the MaxMind API. This means that local binary databases can also be queried, which is far faster and more robust than web-based equivalents and avoids the privacy concerns associated with transmitting users' IP addresses externally.

The MaxMind API requires a paid or free binary database - one of which, for country-level IP resolution, is included in rgeolocate - and allows you to retrieve the continent, country name or ISO code, region or city name, tzdata-compatible timezone, longitude, latitude or connection type of a particular IP address. Multiple fields can be selected (although which are available depends on the type of database used), and results are returned in a data.frame: 


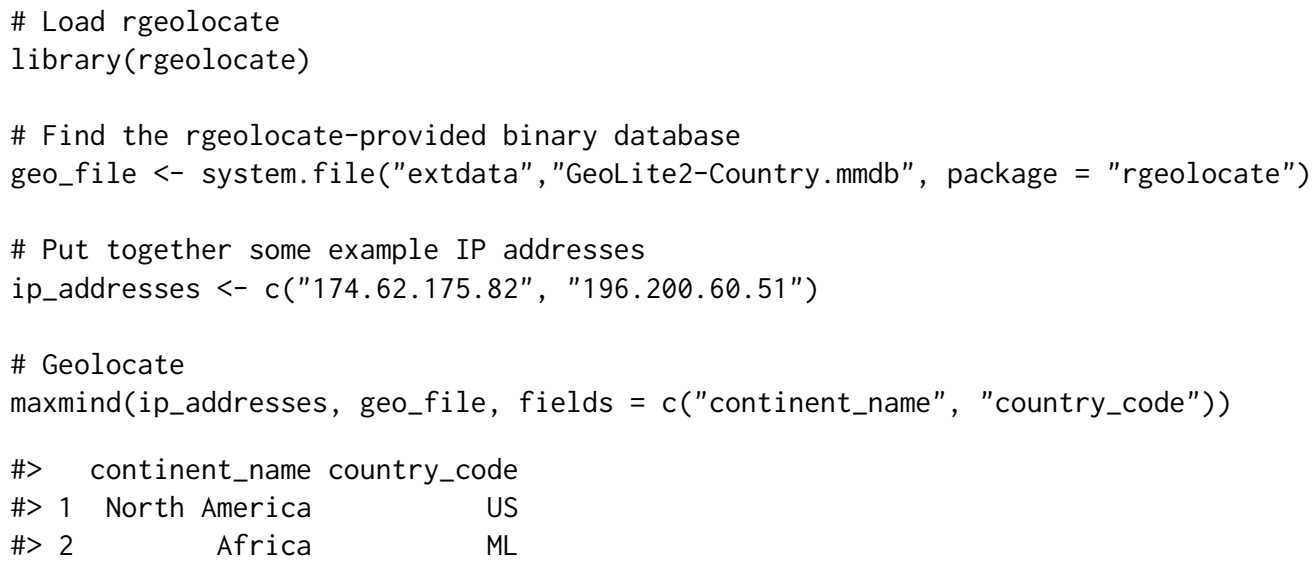

Direct speed comparisons aren't possible, since the functionality it provides is not, to the authors' knowledge, replicated in other $\mathrm{R}$ packages, but it is certainly faster and more secure than internetdependent alternatives.

\section{Conclusions and further work}

In this research article we have demonstrated a set of tools for handling access logs during every stage of the data cleaning pipeline - reading them in with webreadr, decoding, manipulating and extracting value from URLs with urltools, and retrieving geographic metadata from IP addresses with iptools and rgeolocate. We have also demonstrated the dramatic speed improvements in using these tools in preference to existing methods within $\mathrm{R}$.

In combination, this makes for an incredibly powerful set of tools for analysing web data, providing functionality and economies of scale not previously available in $\mathrm{R}$ and making $\mathrm{R}$ a first-class environment for web data analysis. In the introduction, we gave an example of a common workflow for researchers dealing with these sorts of logs: knitting the examples above together, we can see that the tasks have gone from inefficient to efficient (in the cases of data reading and URL manipulation) and from impossible to possible in the case of IP geolocation.

Further work - integrating more sources of geolocation information within rgeolocate, supporting UTF-8 URL decoding within urltools, and increasing IPv6 support in iptools - would make these packages even more useful to Human-Computer Interaction researchers and other specialists who rely on access logs as primary data sources. Possible sources of performance improvements include Even without that functionality, however, this suite of packages is a dramatic improvement upon the status quo.

\section{Acknowledgements}

This paper would not have been possible without the support of Margret Wander, Adam Hyland and Penelope Hopkins, and the copyediting and commentary by Laurent Gatto, Brandon Hurr and Hadley Wickham. In addition, we would like to thank Toby Negrin for inspiring us to write a paper on these packages, rather than just the packages themselves.

The code for the benchmarking included in the figures can be found in the git repository for the paper, and is MIT-licensed.

\section{Bibliography}

Boost C++ Libraries. URL http://www. boost.org. http://www. boost.org. [p363]

T. Berners-Lee, L. Masinter, and M. McCahill. Uniform resource locators (url). RFC 1738, December 1994. URL https://tools.ietf.org/html/rfc3986. [p362]

A. S. Bhingarkar and B. D. Shah. A survey: Securing cloud infrastructure against EDoS attack. In Proceedings of the International Conference on Grid Computing and Applications (GCA), page 16. The Steering Committee of The World Congress in Computer Science, Computer Engineering and Applied Computing (WorldComp), 2015. [p360]

G. Grolemund and H. Wickham. Dates and times made easy with lubridate. Journal of Statistical Software, 40(3):1-25, 2011. URL http://www. jstatsoft. org/v40/i03/. [p363] 
A. Halfaker, O. Keyes, D. Kluver, J. Thebault-Spieker, T. T. Nguyen, K. Shores, A. Uduwage, and M. Warncke-Wang. User session identification based on strong regularities in inter-activity time. CoRR, abs/1411.2878, 2014. URL http://arxiv.org/abs/1411.2878. [p360]

O. Keyes. webreadr: Tools for Reading Formatted Access Log Files, 2015. URL http: //CRAN. R-project. org/package=webreadr. R package version 0.3.0. [p361]

O. Keyes, J. Jacobs, M. Greenaway, and B. Rudis. urltools: Vectorised Tools for URL Handling and Parsing, 2015a. URL http://CRAN. R-project.org/package=urltools. R package version 1.3.2. [p362]

O. Keyes, D. Schmidt, D. Robinson, I. Maxmind, and P. Gloor. rgeolocate: IP Address Geolocation, 2015b. URL http: //CRAN.R-project.org/package=rgeolocate. R package version 0.5.0. [p364]

D. T. Lang and the CRAN Team. XML: Tools for Parsing and Generating XML Within R and S-Plus, 2016. URL https: //CRAN. R-project. org/package=XML. R package version 3.98-1.4. [p362]

D. S. Mendonca. ApacheLogProcessor: Process the Apache Web Server Log Combined Files, 2015. URL http://CRAN. R-project.org/package=webreadr. R package version 0.1.5. [p361]

O. Mersmann. microbenchmark: Accurate Timing Functions, 2014. URL http: //CRAN. R-project. org/ package=microbenchmark. R package version 1.4-2. [p361]

B. Rudis and O. Keyes. iptools: Manipulate, Validate and Resolve IP Addresses, 2015. URL http: //CRAN. Rproject. org/package=iptools. R package version 0.3.0. [p363]

F. Ryckbosch and A. Diwan. Analyzing performance traces using temporal formulas. Software: Practice and Experience, 44(7):777-792, 2014. [p360]

S. W. Sen, H. Ford, D. R. Musicant, M. Graham, O. S. Keyes, and B. Hecht. Barriers to the localness of volunteered geographic information. Proceedings of the 2015 ACM Conference on Human Factors in Computing, 2015. [p364]

H. Wickham. httr: Tools for Working with URLs and HTTP, 2015. URL http: //CRAN. R-project. org/ package=httr. R package version 1.0.0. [p360, 362]

H. Wickham and R. Francois. readr: Read Tabular Data. URL https://github.com/hadley/readr. R package version 0.1.1.9000. [p361]

\author{
Oliver Keyes \\ Rapid7 \\ One Main Street, Penthouse \\ Cambridge, MA, 02142, USA \\ ironholds@gmail.com
}

Bob Rudis

Rapid7

One Main Street, Penthouse

Cambridge, MA, 02142, USA

brudis@rapid7.com

Jay Jacobs

BitSight

Boston, MA

jay@beechplane.com 